\title{
ENSEÑANZA PARA LA COMPRENSIÓN EN ENTORNOS VIRTUALES DE APRENDIZAJE: LA HERRAMIENTA “FORO" Y LA ETAPA EXPLORATORIA. ESTUDIO DE UN CASO EN UN CURSO DE FISIOLOGÍA HUMANA
}

\author{
TEACHING FOR UNDERSTANDING IN VIRTUAL \\ LEARNING ENVIRONMENTS: TOOL "FORUM" AND \\ EXPLORATORY STAGE. CASE STUDY COURSE IN \\ HUMAN PHYSIOLOGY
}

\begin{abstract}
Nazira Píriz Giménez*
nazirapiriz@gmail.com
\end{abstract}

\section{RESUMEN}

La Enseñanza para la Comprensión (EPC) entiende a la comprensión como capacidad de desempeño flexible. Propone "desempeños de comprensión" y sugiere la progresión en categorías: - etapa de exploración, - etapa de investigación guiada; y - proyecto final de síntesis. La modalidad Semipresencial de Profesorado en Uruguay, presenta características que la hacen ideal para el trabajo en un marco de EPC. Este trabajo analiza un foro de discusión de Fisiología humana, como instancia para la presentación de un tópico generativo y el trabajo en la etapa de exploración.

Los resultados muestran su riqueza para el logro de los objetivos propuestos por la EPC a estos efectos.

Palabras clave: Enseñanza para la comprensión, Entornos virtuales de aprendizaje, foros de discusión.

\begin{abstract}
Teaching for Understanding (TfU) regards understanding as a flexible performance competence, which enables comprehension activities to be carried out. It proposes a development through different categories: -investigation stage, -guided investigation stage, and -final synthesis project. The methodology of distant learning in the teaching training course in Uruguay, presents the characteristics that make it ideal to work within the framework of TfU. This work analyses a discussion forum on Human Physiology as an instance for the presentation of a generative topic and the work in the investigation stage.
\end{abstract}

The results show its productivity for the achievement of the TfU's objectives.

Keywords: Teaching for Understanding, Virtual learning environments, learning forums.

* Profesora y tutora de Biofísica y Fisiología humana. IPA, Prof. Semipresencial - CFE. Médica, Mag. Biofísica, Diploma Tutorías virtuales, Doctoranda Educación. Becaria MEC (2014). Píriz y Gelós (2015) Rev. InterCambios, N³; Píriz (2013) Biofísica para la formación del Profesorado, Montevideo: Ediciones Ciencia, 176 p.; Píriz y Perendones (2013) y López, Rattín, Curbelo, Alcaín, Tucci y Píriz (2013), publicaciones del CFE, Píriz (2011) RSEUS №2, 115 - 125. 


\section{INTRODUCCIÓN}

\section{En relación a la Enseñanza para la comprensión:}

La Enseñanza para la Comprensión (EPC) constituye una teoría pedagógica propuesta a fines del siglo pasado por un grupo de investigadores de la Universidad de Harvard, entre quienes se destacan: David Perkins, Martha Stone Wiske, y Tina Blythe. Si bien la EPC recoge algunos elementos propuestos anteriormente por diversos autores constructivistas, se caracteriza fundamentalmente por la introducción de un nuevo concepto de "comprensión". En tal sentido, a diferencia de teorías previas que entienden a la comprensión como una representación mental de algún tipo, la EPC entiende a la "comprensión" como un estado de capacitación que se manifiesta como un desempeño en la realización de actividades de comprensión. Según Perkins, comprender es la habilidad de pensar y actuar con flexibilidad a partir de lo que uno sabe. Comprender un tópico implica entonces la capacidad de desempeño flexible (Perkins, D, 1997).

La EPC propone que desde el diseño de la currícula deben considerarse diversos aspectos, de manera por ejemplo, que los cursos se organicen en torno a temáticas integradoras (tópicos generativos), que posibiliten estrategias heurísticas y estimulen el descubrimiento. "Los temas del currículo deberían no sólo "cubrirse" sino "descubrirse" de modos que alienten la indagación continua..." dice Stone (2006). En tal sentido, los tópicos generativos deben ser temas que reúnan las siguientes características: a) deben estar conectados con múltiples ideas importantes no sólo de la materia en cuestión sino también de las demás materias; b) deben ser auténticos, accesibles e interesantes para los estudiantes; c) deben ser fascinantes e inspiradores para los docentes; y 4) deben ser abordables desde una variedad de ángulos diversos y a través de una serie de materiales curriculares y de las tecnologías disponibles. Los tópicos generativos deben ser "inagotables", y deben generar y estimular la indagación continua.

Resulta claro de lo expuesto, la jerarquización que la EPC hace de la motivación, elemento ya destacado por diversos autores constructivistas como Ausubel (Ausubel, D., 1976). En relación a

$:: 24::$ la motivación, la bibliografía define dos grandes categorías de estilos motivacionales: motivación extrínseca e intrínseca (Carretero, 2004; Díaz Barriga, 2001). La diferencia central entre ambos estilos radica en que mientras la motivación extrínseca se centra en las expectativas y aprobación de terceros, la motivación intrínseca se centra en la tarea en sí misma y la satisfacción personal de aprender. En forma sintética, quienes tienen un predominio intrínseco de la motivación disfrutan del proceso de aprendizaje que viven con autocrítica y consciencia de la necesidad de esfuerzo y dedicación, reconocen el error como natural del proceso, y el "premio" es el propio aprendizaje. La EPC, al proponer tópicos generativos que estimulen a su descubrimiento, alude a la motivación intrínseca, la que puede promoverse a través de este tipo de propuestas. Este enfoque es también compatible con el concepto de aprendizaje significativo y de cognición situada (Ausubel, D., 1976; Díaz Barriga F y Hernández, 2001; Díaz Barriga, F., 2003; Carretero, M., 2004).

En relación a la comprensión, la EPC define cuatro niveles graduales: - nivel de contenido (comprensión ingenua: el estudiante puede realizar actividades reproductivas pero no de comprensión); - nivel de resolución de problemas (comprensión de principiante: hace referencia a problemas cuya resolución provee práctica, "entrena"); - nivel epistémico (comprensión de aprendiz: realiza actividades de comprensión como explicar y justificar); - nivel de investigación (comprensión de maestría: plantea hipótesis, realiza cuestionamientos) (Perkins D, 1997; Stone, 1999).

Por otra parte, la EPC habla de "desempeños de comprensión" como actividades que desarrollan y demuestran comprensión. Ellos incluyen: "explicar, interpretar, analizar, relacionar, comparar y hacer analogías". Paralelamente, los investigadores reconocen la importancia en la progresión de las categorías de desempeño a proponer por los docentes, como forma de que las actividades impliquen un desafío para el estudiante. Se reconocen tres categorías progresivas de desempeños: 1.- Etapa de exploración. Permite que los estudiantes "establezcan conexiones entre el tópico generativo y sus propios intereses y experiencias previos" (Stone, 1999). Esta etapa permite indagar en lo que los estudiantes ya saben, así como en sus intereses. También puede vincularse con la propuesta de Ausubel en tanto jerarquiza el establecimiento de relaciones con los conocimientos previos. 2.Etapa de investigación guiada. En las etapas iniciales, los desempeños pueden ser simples. Pueden 
centrarse las actividades en: "la observación cuidadosa, el registro de datos, el uso de un vocabulario adecuado, la síntesis de diversas fuentes de información en torno a una pregunta". 3.- Proyecto final de síntesis. En esta etapa el estudiante trabaja en forma más independiente demostrando su dominio de las metas de comprensión propuestas.

\section{La modalidad Semipresencial de Profesorado en Uruguay:}

La modalidad Semipresencial de Profesorado dependiente del Consejo de Formación en Educación (CFE), constituye una modalidad que desde hace varios años en nuestro país, permite que estudiantes provenientes de todo el territorio nacional y para quienes no es posible cursar la carrera completa en forma presencial, reciban cursos de asignaturas generales en un Instituto de Formación Docente (IFD) de su departamento y cursos de asignaturas específicas en modalidad semipresencial (en una plataforma virtual y con 3 encuentros presenciales anuales).

Estas características de la modalidad Semipresencial, en particular en lo que refiere a los cursos que se desarrollan en la plataforma virtual, posicionan a la EPC no sólo como una teoría perfectamente compatible y adaptable, sino a nuestro entender, sumamente recomendable. Uno de los argumentos en tal sentido, radica en las características de los encuentros presenciales, instancias de 6 horas de duración y que se repiten 3 veces en todo el año lectivo. En cursos de asignaturas anuales con programas voluminosos en su mayoría, dichos encuentros presenciales pueden constituir un espacio ideal para el trabajo a partir de tópicos generativos no limitando los contenidos temáticos a abordar "clásicas" unidades temáticas, sino que permitan la integración de varias de ellas. De esta manera pueden aprovecharse los encuentros presenciales para el planteo de un tópico generativo y el trabajo en la etapa de exploración, iniciándose también en esta misma instancia la fase de investigación guiada, a continuarse y finalizarse con un proyecto de síntesis, en la plataforma.

Cabe considerar también que el trabajo en la plataforma obliga la participación activa del estudiante, y permite importantes variantes en la propuesta de actividades, que impliquen el desarrollo y el "uso" de una gran diversidad de competencias, habilidades y destrezas, que en el marco de EPC implican diversidad de actividades de comprensión. A modo de ejemplo, actividades tales como el análisis y/o discusión de artículos ó de recursos informáticos (animaciones, simulaciones, etc.), elaboración de materiales diversos, trabajos en proyectos, entre otras actividades, aproximan al estudiante al "aprender a ser y a hacer", tan valorado en los últimos tiempos. Como valor agregado, los entornos virtuales son particularmente propicios para el trabajo en equipo, otra competencia cada vez más jerarquizada en la formación de profesionales.

Por otra parte, un trabajo basado en el modelo de EPC no limita la presentación de tópicos generativos a los encuentros presenciales, sino que precisamente por su reducido número pueden ser necesarias otras instancias. En nuestra opinión, los foros de discusión constituyen la instancia ideal para su planteo en la plataforma virtual. Los foros de discusión hacen posible el intercambio de significados entre estudiantes y la construcción colectiva del conocimiento en un trabajo colaborativo (Bruffee, 1999; Dillenblurg, 1999).

La presentación de un tópico generativo en un foro de discusión y su trabajo en una primera etapa exploratoria, presenta la ventaja adicional de que el estudiante puede elegir de acuerdo a sus intereses e inquietudes los aspectos a indagar en dicha exploración, con una participación natural, sin presiones, en el que la motivación es el motor fundamental de su contribución. Adicionalmente se favorece la colaboración y el intercambio de inquietudes con compañeros.

El rol del tutor en entornos virtuales a modo de orientador y "guía desde el lado" (Tardif, 1998), requiere de un estudiante activo, capaz de realizar actividades de comprensión y no meramente de reproducción.

Como hemos propuesto anteriormente (Píriz Giménez N, 2011), es recomendable que el diseño de actividades a realizar en foros de discusión, considere que dichas actividades no se limiten a la solicitud de definiciones, relatos, o descripciones, sino que sean formuladas de manera de generar cuestionamientos que posibiliten el intercambio de ideas. De esta manera se minimizan las actividades de reproducción favoreciéndose niveles superiores de comprensión. 


\section{PROPUESTA METODOLÓGICA}

Este trabajo consiste en el estudio de un caso en que se analiza un foro de discusión. En dicho foro se propone una actividad que consiste en la presentación de un tópico generativo. El objetivo del presente trabajo es valorar la adecuación de la propuesta para el trabajo en una primera etapa de exploración enmarcada en EPC.

El foro analizado corresponde a la asignatura Fisiología humana de la especialidad Ciencias biológicas de Profesorado Semipresencial, y tuvo lugar en el año lectivo 2012. La consigna de la actividad propuesta para dicho foro fue la siguiente: "Taquicardia, aumento en la presión arterial, midriasis, entre otros, son cambios que experimentan las personas cuando se enamoran. Te proponemos discutir con tus compañeros qué fundamento científico tiene la asociación popular entre corazón y sentimientos". Cabe aclarar que se trata de una propuesta compleja en tanto relata cambios macroscópicos estructurales y funcionales, cuya explicación requiere "viajar" dialécticamente de niveles subcelulares y moleculares a niveles sistémicos de organización biológica. Adicionalmente, su abordaje implica profundizar en contenidos de diversas unidades del programa (Fisiología de los Sistemas Nervioso, Endocrino, y Cardiovascular, fundamentalmente), por lo que se trata de una consigna amplia, inagotable y que da libertad y flexibilidad a los estudiantes para indagar de acuerdo a sus intereses. Acorde a esto, se propuso una dinámica de discusión en el que el tutor se abocó a destacar la jerarquía y el interés de los aportes, solicitar aclaraciones, orientar la resolución de algunos cuestionamientos, apoyando y dinamizando la discusión, con mensajes breves y en un número estrictamente necesario para que la discusión esté centrada siempre en el grupo de estudiantes.

\section{RESULTADOS Y DISCUSIÓN}

A continuación se transcriben mensajes publicados por estudiantes en este foro, así como su análisis. Dichos mensajes se enumerarán para facilitar su cita posterior, y se identifican mediante iniciales a

$:: 26::$ los efectos de cuidar el anonimato de sus autores.

Corresponde aclarar que se trata de un foro en el que participaron 9 estudiantes, seleccionándose al menos un mensaje de cada uno para su análisis. El orden de los mensajes fue respetado en su cita, de manera de vislumbrar también cierto hilo conductor en la discusión, evidenciada por la vinculación entre dichos mensajes.

Mensaje 1: EA: "Hago una introducción a este tema que, como las emociones, de sencillo no tiene nada pero es sumamente interesante...Todas las propiedades cardíacas son intensificadas por el sistema nervioso simpático. Efecto cronotrópico: El aumento de la frecuencia cardíaca que produce la estimulación simpática se debe a un aumento del automatismo del nódulo sinusal".

Mensaje 2: MA: "Los padecimientos y goces del amor se esconden, irónicamente, en esa ingente telaraña de nudos y filamentos que llamamos sistema nervioso autónomo. En ese sistema, todo es impulso y oleaje químico... Es el reino del "siento, luego existo", de las atracciones y repulsiones primarias..., es el territorio donde la razón es una intrusa... Es un poético y discutible aporte que me gustó para compartir... Según lo que entendí (espero correcciones y comentarios), el corazón es un efector, que responde ante la estimulación del sistema simpático que se produce ante "situaciones de alarma" provocadas por las diversas emociones (en este caso el enamoramiento)."

Mensaje 3: AR: "con respecto al sistema límbico... también se lo conoce como "cerebro emocional", ya que desempeña un papel fundamental en una amplia gama de emociones como el dolor, el placer, la docilidad, el afecto y la ira... continúo leyendo!!"

Mensaje 4: TP: "la localización concreta de los circuitos cerebrales en cada una de las emociones, adjudicándole gran importancia al sistema límbico el cual agrupa un conjunto de estructuras corticales y subcorticales que estarían relacionadas con la elaboración de la experiencia emocional y el control de una variedad de funciones viscerales".

Mensaje 5: AB: "la "fisiología del amor", aporto algunos datos sobre un neurotransmisor que, según algunos estudios, tendría un papel fundamental en el comportamiento afectivo. Se trata de la feniletilamina (especialmente en su forma 2-feniletilamina, PEA." 
Mensaje 6: AR: "Qué bueno AB!!! muy interesante el aporte !!!”

Mensaje 7: AB: "Estos temas referidos a las emociones y las funciones cognitivas superiores son apasionantes. Igualmente verás que las fuentes que consulté son escasas y poco rigurosas... aprovecho el msj, para comentar que recientemente leí una nota en un diario sobre un estudio donde se observó la dilatación de la pupila (midriasis) para "conocer" las preferencias sexuales de los participantes. Si bien el objetivo del estudio es discutible, lo menciono porque la midriasis se cita en la actividad."

Mensaje 8: NM: "La actividad neural evocada por estos estímulos complejos se transmite desde el encéfalo anterior hasta los núcleos viscerales y motores somáticos a través del hipotálamo y la formación reticular del encéfalo anterior, las estructuras principales que condicionan la expresión del comportamiento emocional... es un tema muy interesante y que atrapa ya que trabajamos en temas que nos involucran y a veces nos identifican."

Mensaje 9: SS: "El amor en cambio, es producido ante la secreción de dopamina, oxitocina y vasopresina, son sustancias liberadas por estímulos ante los cuales no hay control.... destaco el aula de la que obtuve la información ... http://www... no solo porque me divertí mucho con la clase, sino que muestra claramente 5 hormonas diferentes y que los 2 tipos de sentimientos se dan por un control diferente es decir, hay como controlar la pasión pero no el amor y además muestra que es posible disminuir la secreción de dopamina ante una situación de estrés (como el dolor)."

Mensaje 10: DM: "Según Cannon, la expresión de las emociones se debe a la activación de neuronas talámicas, que derivará en activación de músculos y vísceras y en el envío de información hacia la corteza... enamorarse es precioso y el que nunca halla sentido eso debe tener algún mecanismo alterado o le faltará feniletilamina o algún otro ingrediente. je!"

Mensaje 11: SM: "Una vez superado los primeros estadios del agrado "físico y químico" la oxitocina es también la implicada en la perdurabilidad del amor, que diferentes estudios estiman entre dieciocho meses y tres años. En general, suele atribuirse el enamoramiento a la feniltelitamina y la serotonina, por sus efectos paroxísticos, y a la oxitocina el amor compañero, por sus acciones narcotizantes... a modo de conclusión: "el amor es la más fuerte de las pasiones, porque ataca al mismo tiempo a la cabeza, al cuerpo y al corazón". Voltaire."

\section{DISCUSIÓN DE LOS RESULTADOS}

En primer lugar cabe destacar que la actividad propuesta presenta gran dificultad. Por un lado, porque como tópico generativo, no constituye un "capítulo de libro" al que los estudiantes puedan consultar en un texto, sino que integra contenidos de diversas unidades temáticas del programa, requiriendo además de la consulta de libros de texto, de artículos de divulgación científica, en tanto ninguna fuente por sí misma explica el problema propuesto.

No obstante estas dificultades, y posiblemente por tratarse de una temática que nos involucra tal como plantea uno de los estudiantes, en diversos mensajes ha quedado manifiesta la motivación que ha generado en ellos. De los mensajes transcriptos destacamos: - EA: "Hago una introducción a este tema que, como las emociones, de sencillo no tiene nada pero es sumamente interesante..."; - MA: "Es un poético y discutible aporte que me gustó para compartir...; - AR:“...continúo leyendo!!"; - AR: "Qué bueno AB!!! Muy interesante el aporte!!!"; - AB: "Estos temas referidos a las emociones y las funciones cognitivas superiores son apasionantes."; - NM: "Es un tema muy interesante y que atrapa ya que trabajamos en temas que nos involucran y a veces nos identifican"; SS:- "Destaco el aula de la que obtuve la información ... me divertí mucho con la clase...".

De esta manera, queda evidenciada en primer lugar la motivación que ha generado en los estudiantes la propuesta de este tópico generativo y que los ha llevado a buscar información de diversas fuentes. Destacamos también la actitud de la estudiante SS al compartir el enlace de una clase universitaria sobre el tema, tanto por evidenciar su interés en profundizar en él, como por constituir un gesto colaborativo con sus compañeros. Por otra parte, si bien los mensajes 4 y 5 no expresan explícitamente motivación, destacamos que son mensajes de estudiantes que tuvieron una muy escasa participación en el curso, tratándose el primer caso, de una estudiante que no había participado en foros en los últimos 4 meses del año lectivo. De manera que en este caso, el sólo hecho de participar puede considerarse una señal de interés. Una situación similar se da con 
la estudiante que publica el mensaje 11, quien también participó escasamente en foros previos. Retomaremos este mensaje más adelante en vistas de características particulares.

De esta manera y tal como lo plantea el marco teórico, una de las cualidades esenciales de un tópico generativo radica precisamente en la motivación que debe generar, objetivo que se ha logrado en este caso.

Retomando el mensaje 11, se da una situación muy interesante porque la estudiante establece una relación con contenidos temáticos que claramente no corresponden a la Biología pero que ha aprendido en su vida de estudiante, al citar una frase de Voltaire: “..."El amor es la más fuerte de las pasiones, porque ataca al mismo tiempo a la cabeza, al cuerpo y al corazón"...". De esta manera, entonces, surge otro de los objetivos de la etapa inicial de exploración que el marco teórico propone, como lo es el logro de vinculaciones con conocimientos previos también de otras disciplinas.

Adicionalmente, este foro ha permitido la exploración de nueva terminología científica con la que los estudiantes comienzan a familiarizarse, por lo que la siguiente fase de investigación comienza también a vislumbrarse en esta instancia.

En otra dimensión de análisis referida a los niveles de comprensión, la totalidad de los estudiantes incluyen la transcripción de información (nivel de comprensión ingenuo). Si bien en esta primera etapa exploratoria, es lo esperable, también se vislumbra el trabajo en otros niveles de comprensión. Un ejemplo es la vinculación con la frase de Voltaire ya mencionada, cita en el mensaje 11. Otros son: - En el mensaje 2: “... Según lo que entendí (y espero correcciones y comentarios) el corazón es un efector, que responde ante la estimulación del sistema simpático que se produce ante "situaciones de alarma" provocadas por las diversas emociones (en este caso el enamoramiento)." Dicho mensaje manifiesta una interpretación que además es explicada, por lo que en dicho aporte podemos identificar un nivel de comprensión de principiante (interpretación) y epistémico (explicación). Cabe destacar también en este mensaje la solicitud de correcciones y comentarios por parte de la estudiante, mostrando una actitud abierta a la discusión y de humildad dispuesta a aprender, también acorde a

::28:: un estilo motivacional intrínseco en el que el interés radica en aprender.

Adicionalmente, en el mensaje 7 el estudiante AB plantea: "Aprovecho el msj, para comentar que recientemente leí una nota en un diario sobre un estudio donde se observó la dilatación de la pupila (midriasis) para "conocer" las preferencias sexuales de los participantes. Si bien el objetivo del estudio es discutible, lo menciono porque la midriasis se cita en la actividad.". Nuevamente se establece una relación, en este caso con información tomada de un periódico y por ende de la vida cotidiana. Este mensaje tiene el valor de contribuir a contextualizar el conocimiento (otro ejemplo de actividad propia de una etapa exploratoria), constituyendo además un trabajo intelectual correspondiente a un nivel que supera lo reproductivo.

Resulta también de interés, por tratarse de un foro de discusión en un entorno virtual de aprendizaje, la evidencia de un aprendizaje colaborativo, con mensajes que se vinculan y de los que se desprende un hilo conductor. En tal sentido, el mensaje 1 alude al "Sistema Simpático", que es retomado en el mensaje 2, el que además hace referencia a las "emociones" y al "Sistema límbico", ideas que se retoman en los mensajes 3 y 4 . El mensaje 5 profundiza en lo "afectivo" agregando información en relación a la biología molecular subyacente al fenómeno en estudio. A partir de este mensaje y hasta el último presentado, se "viaja" entre los niveles de organización molecular a sistémico, lo que puede considerarse desde el punto de vista del aprendizaje como un avance y profundización en la discusión, con claras actividades de vinculación. 


\section{CONCLUSIONES}

En primer lugar, destacamos el valor motivacional que tuvo esta propuesta basada en un tópico generativo, que quedó de manifiesto tanto por su verbalización por parte de los estudiantes como por la aparición de participaciones de estudiantes con escasa o nula actividad en el curso hasta ese momento.

En segundo lugar, esta etapa permitió también el establecimiento de relaciones con conocimientos previos tanto de la Biología como ajenos a ella, así como la familiarización con lenguaje técnico, también acorde a lo esperado en esta fase.

El hilo conductor manifiesto en los mensajes citados evidencia el logro de un aprendizaje colaborativo, en el que logró cierta profundización y avance, contribuyendo a la apropiación de terminología científica, también propia de la etapa exploratoria.

En lo que refiere a niveles de comprensión, el predominante fue el primer nivel, no obstante surgieron actividades correspondientes a niveles de principiante y epistémico, como lo son la interpretación y explicación, respectivamente.

Surge también luego de finalizado el foro, la necesidad de profundizar en los contenidos abordados. Según el marco teórico de EPC, seguiría una fase de investigación guiada y posteriormente una fase de proyecto de síntesis. Dichas fases se continuaron en el curso, si bien no son objeto de análisis de este trabajo.

Finalmente, se puede decir que los foros de discusión constituyen una herramienta valiosa para la presentación de tópicos generativos en un marco de EPC, que permiten desarrollar la primera etapa exploratoria promoviendo la motivación, el establecimiento de relaciones con conocimientos previos y la familiarización con la terminología científica, pudiendo además insinuarse la siguiente etapa de investigación guiada mediante la aparición de explicaciones e interpretaciones. Se agrega desde el punto de vista de la Didáctica de la Biología, la ventaja de permitir vincular diversos niveles de organización, así como contextualizar los procesos con hechos de la vida cotidiana. En relación a los niveles de comprensión, si bien en esta etapa predominan las actividades reproductivas, también es posible el avance en otros niveles. Tal como lo propone el marco teórico, es preciso luego de esta fase, la propuesta de nuevas actividades que permitan profundizar en los contenidos en una investigación guiada, así como una etapa final de proyecto de síntesis, que si bien fueron desarrollados en el curso, no fue objeto de análisis de este trabajo.

\section{İNDICE DE FUENTES}

\section{SECUNDARIAS}

\section{Bibliografía}

AUSUBEL, D. (1976): Psicología educativa. Un punto de vista educativo. Mexico: Editorial Trillas. BRUFFEE, KENNETH A. (1999): Collaborative Learning. Higher Education, Interdependence and the Authority of Knowledge. The Johns Hopkins University Press. Baltimore and London.

CARRETERO, M (2004): Constructivismo y Educación. Buenos Aires: Aique.

DÍAZ BARRIGA, F (2003): "Cognición situada y estrategias para el aprendizaje significativo". Revista Electrónica de Investigación Educativa, [en línea] vol.5 No2. [Fecha de consulta 06/07/2012]. Disponible en: http://redie.uabc.mx/index.php/redie/article/view/85/151

DÍAZ BARRIGA, F; HERNÁNDEZ G. (2001): Estrategias docentes para un aprendizaje significativo. Una interpretación constructivista. Mexico: Mc Graw-Hill.

DILLENBLURG, PIERRE (1999): Collaborative Learning. Cognitive and Computacional Approches. Pergamon, Elsevier Science, Oxford.

PERKINS, D. (1997): La escuela inteligente. Del adiestramiento de la memoria a la educación de la mente. Barcelona: Editorial Gedisa. 
PÍRIZ GIMÉNEZ, N (2011): "Emergencia de nuevas competencias en Profesorado Semipresencial y su integración al currículo. Lineamientos de una propuesta". RSEUS N², p 115-125.

STONE WISKE, MARTHA (2006): Enseñar para la comprensión con nuevas tecnologías. Barcelona: Editorial Paidós.

STONE WISKE, MARTHA (1999): La Enseñanza para la Comprensión. Vinculación entre la investigación y la práctica. Barcelona: Editorial Paidós.

TARDIF, J (1998) : Intégrer les nouvelles technologies de l'information. Quel cadre pédagogique? ESF éditeur.

Recibido el 03 de marzo de 2014

Aceptado el 21 de mayo de 2014

$:: 30::$ 